\title{
Efficacy, safety and clinical use of newer basal insulins analogs
}

\begin{abstract}
The aim of insulin therapy in patients with diabetes is to match the endogenous pattern of insulin secretion as closely as possible without causing hypoglycemia. In the last decade, the introduction of insulin analogues has allowed significantly improvement in glycemic control and has facilitated the use of basal/bolus regimens, the most physiological ones until now. There still is room for improvement in the pharmacokinetic/pharmacodynamic profile of basal insulins, and three new products offer this prospect. This article pinpoints the main difficulties regarding insulin therapy in diabetes, the problems with currently available basal insulin analogs followed by a review of the available data on efficacy, safety and clinical use of the new basal insulin analogues degludec, glargine U300 and pegylated lispro. While further study will be required, advances in basal insulin replacement may offer advantages over existing options for starting and progressing insulin strategies in patients with both type 1 and type 2 diabetes.
\end{abstract}

Keywords: basal insulin analogues, deglutec, glargina 300UI, diabetes mellitus
Volume 6 Issue 3 - 2018

\author{
Maria Tereza Martins Ferrari,' Arnaldo \\ Moura Neto ${ }^{2}$ \\ 'Endocrinology Department, Santa Marcelina Hospital, Brazil \\ ${ }^{2}$ Clinical Medicine Department, Faculty of Medical Sciences, \\ University of Campinas, Brazil
}

\begin{abstract}
Correspondence: Arnaldo Moura Neto, Endocrinology Division, Clinical Medicine Department, Faculty of Medical Sciences, University of Campinas Rua Tessalia Vieira de Camargo, I26-Barao Geraldo CEP I3084-97 I/Campinas-Sao Paulo-Brazil, Brazil, Email arnaldo.mouraneto@gmail.com
\end{abstract}

Received: February 26, 2018 | Published: June 05, 2018
Abbreviations: FPG, fasting glucose glucose; HbA1C, glycated haemoglobin; IAsp, insulin Aspart; IDeg, insulin degludec; IDet, insulin detemir; IGla, insulin glargine; Gla-300, 300 units $/ \mathrm{ml}$; Cmax, maximum plasmatic concentration; PK, Pharmacokinetic; PEG, polyethylene glycol; SMPG, self-measured plasma glucose; T1DM, type 1 diabetes; T2DM, type 2 diabetes

\section{Introduction}

Basal insulin analogues have been developed to mimic the action of endogenous insulin secretion in fasting and post-absorptive states. It is an important treatment component for both type 1 (TIDM) and type 2 diabetes (T2DM). However, currently available basal insulins, including Glargine (IGla) and detemir (IDet) have a number of limitations that distance them from the ideal pharmacokinetic (PK) and pharmacodynamic properties of an ideal basal insulin. These limitations include a relatively short half-life that does not consistently allow adequate glycaemic control over a full 24-h period with once-daily dosing. ${ }^{1-3}$ Furthermore, the lack of a flat and stable glucose lowering effect can make titration difficult in an individual subject and increase the hypoglycaemia risk. ${ }^{4,5}$ Ideally, basal insulins should have a duration of action of over $24 \mathrm{~h}$, a flat and peakless PK profile, a stable and reliable rate of absorption and absent inter and intra patient variability. Additionally, the drug must present a low risk of hypoglycaemia, especially for nocturnal and severe episodes. New basal insulins, with an improved phamarcodymanic profile, including prolonged and consistent biological action, lower risk of hypoglycaemia and more flexible dosing schedule, were developed to address these issues with the primary objective of improving longterm glycaemic control and the patient's management with basal evaluated at this time include insulin Degludec (IDeg) and a new formulation of glargine known as Glargin 300 (Gla-300).

\section{The need for better basal insulins}

Despite the development of where $75,5 \%$ of physicians insulin therapy has seen in the last decades and the improved education of patients and their health care providers, the likelihood of achieving good glycaemic control remains discouragingly low. ${ }^{6,7}$ Poor glycaemic control is highly prevalent with $44-63 \%$ of patients not achieving glycaemic targets. ${ }^{8-11}$ Additionally, hypoglycaemia (or fear of it) is widely perceived as a major limiting aspect in the success of insulin therapy. ${ }^{12}$ In one study, $25 \%$ of patients reduced their insulin dose in response to a non-severe hypoglycaemia event. ${ }^{12,13}$ Aside from the impact in regimen adherence, the fear of hypoglycemia can also delay practitioners from initiating insulin or intensifying therapy, as illustrated in the Global Attitudes of Patients and Physicians in Insulin Therapy (GAPP) study that $75,5 \%$ of physicians admitted they would treat their patients more aggressively if there was no increased risk of hypoglycaemia. ${ }^{14}$ If one considers the results of epidemiological studies and clinical trials, which suggest that hypoglycaemia has a negative impact on cardiovascular morbidity and mortality, this delay could be even longer. ${ }^{15-19}$ Another important factor, which might be under-appreciated, is variable glucose readings causing difficulties in dose adjustment. Not only is glucose variability a major confounding factor in disease management but it also has direct prognostic consequences and is increasingly recognized as an informative parameter in diabetes management. The role of insulininduced glucose variability is particularly important for basal insulins because of their prolonged absorption from high-dose depots. ${ }^{20}$ In clinical trials, the currently available basal insulin analogues have often shown excellent overall glycaemic control with low incidence of hypoglycaemia, especially nocturnal. ${ }^{21-25}$ In routine clinical practice, however, insulin analogue-treated patients often do not achieve good glycaemic control. This might reflect a suboptimal use and pharmacokinetic profile of these products. Therefore, there is room for improvement and it is absolutely welcome.

\section{Insulin degludec}

Insulin Degludec (IDeg) is based on human insulin, modified by removal of the B30 threonine amino acid residue, and acylating the now DesB30 human insulin at the e-amino group of LysB29 with hexadecandioic acid via a g-Lglutamic acid spacer. ${ }^{26,27}$ IDeg is formulated in the presence of phenol and zinc to create a solution of di- 
hexamers. Following subcutaneous (SC) injection and the dispersion of phenol, the di-hexamers self-associate to form a stable depot of multi-hexamer chains at the injection site. The subsequent diffusion of zinc from the multi-hexamers results in gradual dissociation of these chains into readily-absorbed IDeg monomers, providing a slow and continuous delivery of IDeg into the circulation. ${ }^{26}$ In addition, IDeg can bind strongly but reversibly to albumin via its fatty diacid side chain, resulting in plasma protein binding of more than $99 \%{ }^{28}$ These mechanisms allow a very slow and flat absorption. Upon IDeg injection, insulin concentrations rise immediately but slightly, achieving maximum plasmatic concentration (Cmax) in $10-12 \mathrm{~h}$, with the mean terminal half-life longer than $25 \mathrm{~h} .{ }^{29-32}$ The duration of action at steady state exceeds $42 \mathrm{~h} .{ }^{29}$ Data show that in individuals with T1DM, IDeg concentrations increased over the first few days of treatment before reaching a plateau. Thereafter, IDeg concentration was unchanged from day to day. ${ }^{33}$ Due to the prolonged pharmacodynamics of IDeg, under steady-state conditions the overlapping of daily injections results in less variability in the glucose lowering effect. ${ }^{34,35}$ In patients with T1DM, it has been shown that the day-to-day, within-subject variability in glucose lowering effect is four times less with IDeg compared with IGlar, theoretically translating into more predictable glycaemic behavior. ${ }^{33}$ The clinical benefits arising from these properties have been verified in a large clinical trial (BEGIN 1) comprising over 11.000 patients with T1DM and T2DM in more than 40 countries (Table 1). Nine phase 3a randomized, controlled, and open label trial with IDeg were designed as treat-to-target trials looking for a fasting blood glucose level target of $70-90 \mathrm{mg} / \mathrm{dl}(4-5 \mathrm{mmol} / \mathrm{L})$. As a result of this method, a comparison can be made in terms of differences in end points such as hypoglycaemia, but not glycated hemoglobin (HbA1C). ${ }^{37-43}$ Indeed, there is another study to evaluate the cardiovascular safety of IDeg in T2DM individuals at high risk of events (DegludEc cardioVascular OuTcomEs trial-DEVOTE trial). ${ }^{44,45}$ This study demonstrated that IDeg was noninferior to glargine in terms of the incidence of cardiovascular events and superior with regard to hypoglycemia risk.

IDeg is available in two formulations: $100 \mathrm{U} / \mathrm{mL}$ (U100) and 200 $\mathrm{U} / \mathrm{mL}$ (U200), with the later designed to allow the administration of up 160 units of IDeg in a single injection. ${ }^{46}$ Another pharmacological property of IDeg is that it can be combined with the rapid-acting insulin analogue Aspart (IAsp) without altering the properties of either components. Insulin degludec/insulin aspart (IDegAsp), comprised of $70 \%$ IDeg and 30\% IAsp. ${ }^{47}$ IDegAsp can be administered once or twice daily with the main meal(s) ${ }^{48,49}$ IDeg can also be combined with liraglutide in IDegLira, a novel, once-daily, fixed ratio combination therapy (one step of IDegLira comprises $1 \mathrm{U}$ of IDeg and $0.036 \mathrm{mg}$ of liraglutide).${ }^{50}$ IDegLira combines and preserves two complementary modes of action addressing the multiple underlying pathophysiological defects in T2DM. ${ }^{51}$ Phase 3 trials (Dual I-V) show that IDegLira leads to effective glycaemic control via reductions in $\mathrm{HbAlc}$, fasting glucose (FPG) and postprandial glucose throughout the day. It is also associated with a significant reduction in body weight vs glargine and IDeg alone; lower risk of confirmed hypoglycaemia and improved glycemic control. ${ }^{52-55}$ The pharmacokinetic characteristics of IDeg are preserved in adult subjects with different degrees of renal impairment. Haemodialysis did not affect the pharmacokinetic profile of IDeg in patients with end-stage renal disease.$^{56}$ In addition, the PK properties of IDeg are preserved in subjects with impaired hepatic function. ${ }^{57}$ The safety and efficacy of IDeg in children and adolescents under the age of 18 has been established. ${ }^{58,59}$

\section{Efficacy of insulin degludec in subjects with type I diabetes}

Studies in subjects with T1DM have shown that IDeg is noninferior to IGlar in terms of mean reduction in HbA1c concentrations and FPG levels. ${ }^{36-38}$ In one basal-bolus trial (IDeg vs IGlar), mean daily basal, daily bolus and total daily doses of insulin were significantly reduced by $14 \%(p<0.0001), 10 \%(p=0,016)$ and $11 \%(p<0.0001)$, respectively, in IDeg group compared with IGlar in the end of trial. ${ }^{37}$

A pre-planned meta-analysis examining hypoglycaemia rates compared IGlar across the phase IIIa program and showed no statistically significant differences in rates of overall confirmed hypoglycaemia (estimated rate ratio (ERR) $1.10,95 \%$ CI 0.96-1.26). The reduction in nocturnal confirmed hypoglycaemia (ERR 0.83, $95 \%$ CI $0.69-1.00$ ) nearly reached statistical significance. ${ }^{60}$ The ERR in nocturnal hypoglycemic episodes reached statistical significance in the maintenance period (ERR 0.75; 95\% IC 0.60-0.94). ${ }^{60}$

\section{Efficacy of insulin degludec in subjects with type 2 diabetes}

Studies in subjects with T2DM confirmed that IDeg is non-inferior to IGlar in terms of reducing HbA1c levels in both insulin-naïve ${ }^{38-61}$ and insulin-treated patients. ${ }^{41}$ There was a trend towards lower FPG concentrations with IDeg compared to IGlar in five trials involving patients with T2DM, ${ }^{38-41,60}$ among which three reached statistical significance. ${ }^{39-41}$ The same pre-planned meta-analysis examining hypoglycaemia rates comparing IDeg with IGlar across the phase IIIa programme demonstrated a $17 \%$ reduction in episodes of overall confirmed hypoglycaemia (ERR $0.83,95 \% 0.74-0.94$ ) and a $32 \%$ reduction in nocturnal confirmed hypoglycemia (ERR 0.68 , 95\% CI 0.57-0.82) during the entire treatment period in subjects with T2DM. ${ }^{60}$ A separate meta-analysis of phase III trial data, focusing on hypoglycaemia rates in elderly patients with T1DM and T2DM, also reported significant reductions in overall and nocturnal hypoglycaemia with IDeg compared with IGlar. ${ }^{63}$

\section{Clinical use of insulin degludec}

When switching patients from other insulins to IDeg, healthcare professionals will need to manage the brief period between the loss of the previous basal insulin's action and attainment of a steady state of IDeg. During this period, patients may observe higher blood glucose levels for 3-5 days and this possibility should be discussed with the patient prior to the switch. In addition, adjustments in other glucoselowering treatments may be required. ${ }^{29}$ Similar to IGlar, IDeg should be administered once daily, preferably at the same time every day. However, due to the ultra-long duration of action (42h) and reduced within-subject variability, IDeg offers the potential for a more flexible dose time window. On occasions, when administration at the same time of the day is not possible, IDeg allows flexibility in the timing of dose administration provided a minimum of $8 \mathrm{~h}$ between injections is ensured. ${ }^{29,32}$ This is supported by two treat-to-target, randomized studies where extreme dosing intervals of $8-40 \mathrm{~h}$ were used in subjects with T1DM and T2DM over treatment duration of 26-52 weeks. ${ }^{38,42}$ The large variation in the injection time of IDeg did not compromise its efficacy or safety compared to IGlar taken at the same time every day. ${ }^{38,42}$ Treatment satisfaction with IDeg in patients with T1DM and T2DM and recurrent hypoglycaemia has been addressed in early, real-world observational studies. Switching patients with frequent hypoglycaemia to IDeg has been associated with a reduction in the 
frequency of hypoglycaemia events, lower hbAlc and improved treatment satisfaction. ${ }^{29,64}$ A meta-analysis has demonstrated a significantly higher score on overall physical component with IDeg compared to IGlar. ${ }^{65}$ Several studies have demonstrated the costeffectiveness of IDeg compared to IGlar. This is a result of the lower rates of hypoglycaemia and its costs, reduced insulin dose requirement, improved patient adherence to the treatment regimen and greater quality of life observed with IDeg. ${ }^{63,66-69}$ The benefit of the lower risk of hypoglycemia can be expressed in terms of numbers needed to treat. In T2DM insulin-nave, for every 100 people treated with IDeg instead of IGla for 1 year, 50 confirmed hypoglycemic episodes (of which 20 are nocturnal) and two severe episodes will be avoided. In basal-bolus-treated T2DM, for every 100 people treated with IDeg instead of IGla for 1 year, 326 confirmed hypoglycemic episodes (of which 71 are nocturnal) will be avoided. In T1DM, for every 100 subjects treated with IDeg instead of IGla for 1 year, 130 nocturnal confirmed episodes will be avoided once the initial titration phase has been completed..$^{59,70}$

\section{Insulin glargine 300 units $/ \mathrm{ml}$}

Insulin glargine 100 units/ml (Gla-100) has become a standard of care in diabetes treatment over the last decade, providing up to 24-h basal insulin coverage after once-daily subcutaneous injection for many people with diabetes, with a well-established efficacy and safety profile. ${ }^{71}$ New insulin glargine 300 units/ml (Gla-300) is a basal insulin that provides the same number of units as Gla-100 in a third of the volume. It is hypothesized that this modifies the surface area of the subcutaneous depot influencing the re-dissolution rate. ${ }^{72}$ One consequence of this is that Gla-300 appears to have better pharmacokinetic and pharmacodynamic profiles. ${ }^{72,73}$ The prolonged duration of action of Gla-300 compared with Gla-100 may also be particularly beneficial to people who currently require twice-daily injections of basal insulin. Clamp studies have demonstrated stable, constant activity over $24 \mathrm{~h}$ after dosing, with a slow decline thereafter, and with measurable activity until the end of the clamp. ${ }^{72}$ An additional study of Gla-300 at steady state found that it provided an evenly distributed coverage for $24 \mathrm{~h}$, with low within-day variability. ${ }^{74}$ The clinical efficacy of U300 was evaluated in six Phase III, multicentre, randomized, open-label, parallel-group, 6-month clinical trials known as the EDITION series. The EDITION series evaluated the efficacy and safety of Gla-300 in diverse populations of people with diabetes (Table 2). The primary endpoint in each of these studies was the change in $\mathrm{HbA} 1 \mathrm{c}$ from baseline to month $6 .^{75-80}$ The first main secondary endpoint in EDITION 1, 2, and 3 was the percentage of participants experiencing $\geq 1$ confirmed [blood glucose $\leq 3.9 \mathrm{mmol} / 1$ $(\leq 70 \mathrm{mg} / \mathrm{dl})]$ or severe nocturnal (00:00-05:59 hours) hypoglycemic event from week 9 to month $6 .{ }^{75-77}$

\section{Efficacy of Glargine $300 \mathrm{U}$ in subjects with type I diabetes}

The study EDITION 4 compared the efficacy, tolerability, and safety of Gla-300 compared to Gla-100 in a population of people with T1DM and investigated whether these outcomes differed when injections were given in the morning or evening. Gla-300 was shown to be non-inferior to Gla-100 for HbAlc reduction from baseline. ${ }^{78}$ Similar results were found for morning and evening injection times and for pre-breakfast self-measured plasma glucose (SMPG) overall. Results were also similar for Gla-300 when morning and evening injection time was compared, including overlapping 8-point SMPG profiles. Hypoglycemia did not differ, except for the first 8 weeks of the study, when nocturnal confirmed or severe hypoglycemia was lower with Gla-300 (risk ratio 0.69 [95\% CI $0.53-0.91]$ ). ${ }^{80}$ There was a greater increase in total insulin dose with Gla-300 compared with Gla-100 (change from baseline: 0.19 units $/ \mathrm{kg}$ vs 0.10 units $/ \mathrm{kg}$ ); weight gain with Gla-300 was significantly less compared with Gla100 [difference: $-0.56 \mathrm{~kg}(95 \%$ [CI-1.09 to -0.03 ]; $p=0.037){ }^{80}$ These findings differ from the type 1 diabetes study in Japan, where Gla-300 did show an advantage for nocturnal hypoglycemia. ${ }^{74}$ In EDITION JP 1 the annualized rate of confirmed $(\leq 3.9 \mathrm{mmol} / \mathrm{l})$ or severe hypoglycemic events was $34 \%$ lower with Gla-300 than with Gla100 at night [rate ratio $0.66(95 \%$ CI $0.48-0.92)$ ] and $20 \%$ lower at any time of day $(24 \mathrm{~h}$; rate ratio 0.80 [95\% CI $0.65-0.98]){ }^{78}$

\section{Efficacy of Glargin $300 \mathrm{U}$ in subjects with type 2 diabetes}

The EDITION 1, 2 and 3 studies showed that Gla-300 and Gla100 provided comparable glycaemic control, alongside a lower risk of hypoglycaemia with Gla-300, over 6 months. This is attributed to the more constant and prolonged pharmacokinetic and pharmacodynamic profile of Gla-300 compared with Gla-100. ${ }^{74-77}$ The EDITION 1 clinical trial enrolled patients with T2DM who had inadequate glycaemic control on basal and mealtime insulin. ${ }^{74}$ Patients were randomized $1: 1$ to receive treatment in the evening with either Gla-300 or Gla-100. All patients continued to receive mealtime insulin, and basal insulin was titrated to achieve a FPG level of $80-100 \mathrm{mg} / \mathrm{dl}$; no significant differences were observed for $\mathrm{HbA} 1 \mathrm{c}, \mathrm{FPG}$, eight-point self-monitored glucose profiles and pre-injection blood glucose levels. With regard to the occurrence of confirmed or severe hypoglycaemia at any time, Gla-300 was similar to Gla-100 (81.9\% vs. $87.8 \%$; RR 0.93 [0.88$0.98]$ ), but for nocturnal events, U300 was superior to U100 (44.6\% vs. $57.5 \%$; RR 0.78 [0.68-0.89]). ${ }^{74}$ Following the blinded 6-month treatment period, all patients continued to receive U300 or U100 in a 6-month open-label extension. ${ }^{81}$ Over the 12 -month treatment period, the mean basal insulin dose was higher in the Gla-300 group (1.03 vs $0.90 \mathrm{U} / \mathrm{kg}$ ). Improvements in hyperglycemic control relative to baseline were maintained at 12 months in both treatment groups, but Gla-300 produced numerically greater reductions in $\mathrm{HbA1c}(+0.17 \%)$ and FPG $(+0.34 \mathrm{mmol} / \mathrm{l})$. The proportion of patients who experienced at least one confirmed or severe hypoglycemic episode at any time of day was $85.9 \%$ for Gla-300 and $91.5 \%$ for Gla-100 (RR 0.94 [0.89$0.99])$, with a greater difference between the two groups for nocturnal hypoglycemic episodes (54.5\% vs $64.7 \%$; RR 0.84 [0.75-0.94]; p = 0.007). ${ }^{81}$

In the EDITION 2 clinical trial, Gla-300 was compared with Gla100 in patients with T2DM inadequately controlled by basal insulin and oral agents. ${ }^{76}$ The initial 6-month treatment period was followed by a 6-month open-label extension and the entire 12-month treatment period was completed by $315(78 \%)$ and $314(77 \%)$ of patients in the Gla-300 and the Gla-100 groups, respectively. ${ }^{82}$ Improvements in glycaemic control at 6 months were maintained at 12 months in both groups. Of note, mean weight gain was significantly lower with Gla-300 than Gla-100 (0.42 vs $1.14 \mathrm{~kg}$; p <0.0091). At 12 months, the proportion of patients with at least one confirmed or severe hypoglycemic event, at any time and during the night, was 78.4 and $37.5 \%$, respectively, in the Gla-300group, and 82.0 and $44.6 \%$, respectively, in the Gla-100 group. These data were used to calculate the 12-month per-patient rate of confirmed or severe nocturnal hypoglycemic events, demonstrating a $37 \%$ reduction in 
risk with Gla-300 versus Gla-100 (RR 0.63 [0.42-0.96]). ${ }^{82}$ The safety and efficacy of Gla-300 versus Gla-100 were further investigated in the EDITION 3 trial in insulin-naive patients with inadequately controlled T2DM. ${ }^{77}$ Mean changes in HbAlc were similar for the two insulin formulations, with a decrease of $1.42 \%$ for Gla-300 and $1.46 \%$ for Gla-100. The frequency of patients who reported at least one confirmed or severe hypoglycemic event at any time was 46.2 and $52.5 \%$ for Gla-300 and Gla-100 (RR 0.88 [0.77-1.61]), respectively, while at least one nocturnal event occurred in 17.9 and $23.5 \%$ for Gla300 and Gla-100 (RR 0.78 [0.59-0.99]), respectively. ${ }^{77}$ A patient-level meta-analysis of the data from the EDITION studies with individuals with type 2 diabetes (EDITION 1, 2, and 3) was conducted to assess glycaemic control and hypoglycaemia over 6 months in a large heterogeneous population (Gla-300: $n=1247$; Gla-100: $n=1249$ ) ${ }^{81}$ Overall, mean (SEM) change in HbA1c was similar in the Gla-300 and Gla-100 groups $[-1.02(0.03) \%$ vs $-1.02(0.03) \%$, respectively]. The proportion of individuals experiencing $\geq 1$ confirmed or severe nocturnal hypoglycemic event was significantly lower for Gla-300 than Gla-100 over the entire 6-month period (RR 0.75 [0.68-0.83]). ${ }^{81}$ The percentage of individuals who experienced $\geq 1$ confirmed or severe hypoglycemic event at any time of day was also significantly lower for Gla-300 than Gla-100 over the entire 6-month period (RR $0.91[0.87-0.9]) \cdot{ }^{83}$ In this pooled analysis of EDITION 1, 2 and 3, over the 6-month treatment period, the number of participants who would need to be treated with Gla-300 in order to prevent one confirmed $[\leq$ $3.9 \mathrm{mmol} / 1(\leq 70 \mathrm{mg} / \mathrm{dl})]$ or severe hypoglycemic event compared with treatment with Gla-100 was $16 .^{83}$

\section{Clinical use of insulin Glargine $300 \mathrm{UI} / \mathrm{ml}$}

Gla-300 offers a potential advantage for those patients requiring large doses of insulin, in which absorption can be an issue. Gla-300 is three times as concentrated as other basal insulins and this may allow patients to administer a great number of units per injection. ${ }^{84}$ For those with type 1 diabetes, the recommended starting dose of Gla300 is calculated by taking the total daily insulin dose, estimated by $0.2-0.4$ units $/ \mathrm{kg}$ of body weight, and giving one third to one half of the total daily dose calculated. For those T2DM insulin-naive patients, it is reasonable to initiate Gla-300 at 0.2 units $/ \mathrm{kg}$ of body weight once daily ${ }^{85}$ When converting a patient who is already receiving intermediate or long-acting insulin once daily, the dose of Gla-300 would be a one-to-one conversion and given once daily. ${ }^{85}$ Assessing steady-state conditions based on a terminal half-life of $\square 19 \mathrm{~h}$, insulin glargine blood concentrations are estimated to achieve steady state after 3 to 4 days with Gla-300. So, it is recommended that the dose of Gla-300 should not be titrated more frequently than every $3-4$ days ${ }^{86}$ The results from the EDITION trials showed that by the end of the studies a higher daily insulin dose of Gla-300 was needed compared with Gla-100.The reason for the larger doses with Gla-300 is still unclear and has been postulated to be attributable to longer residence time in the subcutaneous tissue leading to an increase in enzymatic inactivation by tissue peptidases. ${ }^{83}$ This increase in daily dose with Gla-300 would need to be considered if switching from Gla-300 to another long-acting insulin..$^{73}$

\section{Conclusion}

It is remarkable that after almost one century, the hormone insulin continues to inspire molecular innovation and development. Basal insulins form the cornerstone of therapy for type 1 diabetes and many treatment regimens for patients with type 2 diabetes. Comparing with the first-generation insulin analogs, the new basal insulins offer the possibility for a simpler titration algorithm, the potential for a more flexible interval to accommodate different lifestyles and a reduced incidence of hypoglycaemia, especially at night. Nevertheless, for the majority of insulin-requiring patients, whether the need for modern insulins is greater than that to improve behavioral tactics and education in the use of them remains unanswered. On the one hand, well-designed phase 3 studies are essential for regulatory review, but they are not usually sufficient to guide clinicians in the use of new therapies. More effort to identify subgroups with the most favorable benefit-to-risk profiles, both before and after approval for clinical use, would greatly assist clinical providers. On the other hand, new technologies per se are certainly insufficient to ensure the goals related to control of diabetes are achieved. Support from healthcare professionals is all-important, highlighting the importance of discussion with the patient and individualization of treatment. A wise mixture between development in medicine and patient education would bring better long-term outcomes.

\section{Acknowledgments}

None.

\section{Conflict of interest}

The Author declares there is no conflict of interest.

\section{References}

1. Heise T, Meneghini LF. Insulin stacking versus therapeutic accumulation: understanding the differences. Endocr Pract. 2014;20(1):75-83.

2. Arnolds S, Kuglin B, Kapitza C, et al. How pharmacokinetic and pharmacodynamic principles pave the way for optimal basal insulin therapy in type 2 diabetes. Int J Clin Pract. 2010;64(10):1415-1424.

3. Sheldon B, Russell Jones D, Wright J. Insulin analogues: an example of applied medical science. Diabetes Obes Metab. 2009;11(1):5-19.

4. Heise T, Nosek L, Ronn BB, et al. Lower within-subject variability of insulin detemir in comparison to $\mathrm{NPH}$ insulin and insulin glargine in people with type 1 diabetes. Diabetes.2004;53(6):1614-1620.

5. Klein O, Lynge J, Endahl L, et al. Albumin-bound basal insulin analogues (insulin detemir and NN344): comparable time-action profiles but less variability than insulin glargine in type 2 diabetes. Diabetes Obes Metab. 2007;9(3):290-299.

6. Inzucchi SE, Bergenstal RM, Buse JB, et al. Management of hyperglycaemia in type 2 diabetes: a patient-centered approach. Position statement of the American Diabetes Association (ADA) and the European Association for the Study of Diabetes (EASD). Diabetologia. 2012;35(6):1364-1379.

7. Vaag A, Lund SS. Insulin initiation in patients with type 2 diabetes mellitus: treatment guidelines, clinical evidence and patterns of use of basal vs premixed insulin analogues. Eur J Endocrinol. 2012;166(2):159170 .

8. Chan JC, Gagliardino JJ, Baik SH, et al. Multifaceted determinants for achieving glycemic control: the International Diabetes Management Practice Study (IDMPS). Diabetes Care. 2009;32(2):227-233.

9. Hoerger TJ, Segel JE, Gregg EW, et al. Is glycemic control improving in U.S. adults? Diabetes Care. 2008;31(1):81-86.

10. Liebl A, Mata M, Eschwege E. Evaluation of risk factors for development of complications in type II diabetes in Europe. Diabetologia. 2002;45(7):S23-S28. 
11. Lopez Stewart G, Tambascia M, Rosas Guzman J, et al. Control of type 2 diabetes mellitus among general practitioners in private practice in nine countries of Latin America. Rev Panam Salud Publica. 2007; 22(1):12-20.

12. Brod M, Christensen T, Thomsen TL, et al. The impact of non-severe hypoglycemic events on work productivity and diabetes management. Value Health. 2011;14(5):665-671.

13. Brod M, Christensen T, Bushnell DM. Impact of nocturnal hypoglycemic events on diabetes management, sleep quality, and next-day function: results from a four-country survey. J Med Econ. 2012;15(1):77-86.

14. Peyrot M, Barnett AH, Meneghini LF, et al. Insulin adherence behaviours and barriers in the multinational Global Attitudes of Patients and Physicians in Insulin Therapy study. Diabet Med. 2012;29(5):682-689.

15. Monami M, Adalsteinsson JE, Desideri CM, et al. Fasting and postprandial glucose and diabetic complication. A meta-analysis. Nutr Metab Cardiovasc Dis. 2013;23(7):591-598.

16. Yakubovich N, Gerstein HC. Serious cardiovascular outcomes in diabetes: the role of hypoglycemia. Circulation. 2011;123(3):342-348.

17. Zoungas S, Patel A, Chalmers J, et al. Severe hypoglycemia and risks of vascular events and death. $N$ Engl J Med. 2010;363:1410-1418.

18. Gerstein HC, Miller ME, Byington RP, et al. Effects of intensive glucose lowering in type 2 diabetes. $N$ Engl J Med. 2008;358(24):2545-2559.

19. Frier BM, Schernthaner G, Heller SR. Hypoglycemia and cardiovascular risks. Diabetes Care. 2011;34(Suppl 2):S132-S137.

20. Vora J, Heise T. Variability of glucose-lowering effect as a limiting factor in optimizing basal insulin therapy: a review. Diabetes Obes Metab. 2013:15(8):701-712.

21. De Vries JH, Nattrass M, Pieber TR. Refining basal insulin therapy: what have we learned in the age of analogues? Diabetes Metab Res Rev 2007;23(6):441-454.

22. Blonde L, Merilainen M, Karwe V, et al. Patient-directed titration for achieving glycaemic goals using a once-daily basal insulin analogue: an assessment of two different fasting plasma glucose targets-the TITRATE study. Diabetes Obes Metab. 2009;11(6):623-631.

23. Meneghini L, Liebl A, Abrahamson MJ. Insulin detemir: a historical perspective on a modern basal insulin analogue. Prim Care Diabetes. 2010;4(Suppl 1):S31-42.

24. Riddle MC. The treat-to-target trial and related studies. Endocr Pract. 2006;12(Suppl 1):71-79.

25. Riddle MC, Vlajnic A, Zhou R, et al. Baseline HbA1c predicts attainmen of $7.0 \% \mathrm{HbA} 1 \mathrm{c}$ target with structured titration of insulin glargine in type 2 diabetes: a patient-level analysis of 12 studies. Diabetes Obes Metab. 2013;15(9):819-825.

26. Jonassen I, Havelund S, Hoeg Jensen T, et al. Design of the novel protraction mechanism of insulin degludec, an ultra-long-acting basal insulin. Pharm Res. 2012;29(8):2104-2114.

27. Kurtzhals P, Heise T, Strauss HM, et al. Multi-hexamer formation is the underlying basis for the ultra-long glucose-lowering effect of insulin degludec. Diabetologia. 2011;54(suppl 1):S426.

28. Unnikrishnan AG, Bantwal G, Sahay RK. Translating structure to clinical properties of an ideal basal insulin. J Assoc Physicians India. 2014;62(1 Suppl):15-20.

29. Vora J, Cariou B, Evans M, et al. Clinical use of insulin degludec. Diabetes Res Clin Pract. 2015;109(1):19-31.

30. Rendell M. Insulin degludec: a long-acting modern insulin analogue with a predictable pharmacokinetic/pharmacodynamic profile. Drugs Today
(Barc). 2013;49(6):387-397.

31. Tambascia MA, Eliaschewitz FG. Degludec: the new ultra-long insulin analogue. Diabetol Metab Syndr. 2015;7:57.

32. Haahr H, Heise T. A Review of the Pharmacological Properties of Insulin Degludec and Their Clinical Relevance. Clin Pharmacokinet. 2014;53(9):787-800.

33. Coester HV, Heise T, Nosek L, et al. Steady state is reachedwithin 2 to 3 days of once-daily administration of ultra-longactinginsulin degludec. Diabetologia. 2012;36(5)(suppl 1):S47.

34. Heise T, Nosek L, Bottcher SG, et al. Ultra-long-acting insulin degludec has a flat and stable glucose lowering effect in type 2 diabetes. Diabetes Obes Metab. 2012;14(10):944-950.

35. Heise T, Hermanski L, Nosek L, et al. Insulin degludec: four times lower pharmacodynamic variability than insulin glargine under steady-state conditions in type 1 diabetes. Diabetes Obes Metab. 2012;14(9):859-864.

36. Heller S, Buse J, Fisher M, et al. Insulin degludec, an ultra-long acting basal insulin, versus insulin glargine in basal-bolus treatment with mealtime insulin aspart in type 1 diabetes (BEGIN Basal-Bolus Type 1): a phase 3, randomised, open-label, treat-to target non-inferiority trial. Lancet. 2012;379(9825):1489-1497.

37. Mathieu C, Hollander P, Miranda Palma B, et al. Efficacy and safety of insulin degludec in a flexible dosing regimen vs insulin glargine in patients with type 1 diabetes (BEGIN: Flex T1): a 26-week randomized, treat-to-target trial with a 26-week extension. J Clin Endocrinol Metab. 2013;98(3):1154-1162.

38. Zinman B, Philis Tsimikas A, Cariou B, et al. Insulin degludec versus insulin glargine in insulin-naive patients with type 2 diabetes: a 1-year, randomized, treat-to-target trial (BEGIN Once Long). Diabetes Care. 2012;35(12):2464-2471.

39. Gough SC, Bhargava A, Jain R, et al. Low-volume insulin degludec 200 units/mL once daily improves glycemic control similarly to insulin glargine with a low risk of hypoglycemia in insulin-naive patients with type 2 diabetes: a 26-week, randomized, controlled, multinational, treat-to-target trial: the BEGINLOW VOLUME trial. Diabetes Care. 2013;36(9):2536-2542.

40. Gough SC, Bhargava A, Jain R, et al. Low-volume insulin degludec 200 units/mL once daily improves glycemic control similarly to insulin glargine with a low risk of hypoglycemia in insulin-naive patients with type 2 diabetes: a 26-week, randomized, controlled, multinational, treat-to-target trial: the BEGINLOW VOLUME trial. Diabetes Care. 2013;36(9):2536-42.

41. Garber AJ, King AB, Del Prato S, et al. Insulin degludec, an ultralongacting basal insulin, versus insulin glargine in basal bolus treatment with mealtime insulin aspart in type 2 diabetes (BEGIN Basal-Bolus Type 2): a phase 3, randomised, open-label, treat-to-target non-inferiority trial. Lancet. 2012;379(9825):1498-507.

42. Meneghini L, Atkin SL, Gough SC, et al. The efficacy and safety of insulin degludec given in variable once-daily dosing intervals compared with insulin glargine and insulin degludec dosed at the same time daily: a 26-week, randomized, open-label, parallel group, treat-to-target trial in individuals with type 2 diabetes. Diabetes Care. 2013;36(4):858-64.

43. Onishi Y, Iwamoto Y, Yoo SJ, et al. Insulin degludec compared with insulin glargine in insulin-nave patients with type 2 diabetes: a 26week, randomized, controlled, Pan-Asian, treat-to target trial. J Diabetes Investig. 2013;4(6):605-12.

44. Mannucci E, Giannini S, Dicembrini I. Cardiovascular effects of basal insulins. Drug Healthc Patient Saf. 2015;7:113-20.

45. Marso SP, Mc Guire. DEVOTE study group. Efficacy and safety of degludec 
versus glargine in type 2 diabetes. N Engl J Med. 2017;24;377(8):723-732.

46. European Medicines Agency. Insulin degludec. In: Summary of product characteristics; 2013.

47. Heise T, Nosek L, Roepstorff C, et al. Distinct prandial and basal glucose-lowering effects of insulin degludec/insulin aspart (IDegAsp) at steady state in subjects with type 1 diabetes mellitus. Diabetes Ther. 2014;5(1):255-65.

48. European Medicines Agency. Ryzodeg. Summary of product characteristics; 2014.

49. Haahr H, Fita EG, Heise T. A review of insulin degludec/insulin aspart: pharmacokinetic and pharmacodynamic properties and their implications in clinical use. Clin Pharmacokinet. 2017;56(4):339-354.

50. Kumar A. Insulin degludec/liraglutide: innovation-driven combination for advancement in diabetes therapy. Expert Opin Biol Ther. 2014;14(6):86978.

51. Buse JB, Vilsbøll T, Thurman J, et al. Contribution of liraglutide in the fixedratio combination of insulin degludec and liraglutide (IDegLira). Diabetes Care. 2014;37(11):2926-33.

52. Gough SC, Bode B, Woo V, et al. Efficacy and safety of a fixed-ratio combination of insulin degludec and liraglutide (IDegLira) compared with its components given alone: results of a phase 3, open-label, randomised, 26- week, treat-to-target trial in insulin-naïve patients with type 2 diabetes. Lancet Diabetes Endocrinol. 2014;2(11):885-932.

53. Linjawi S, Bode BW, Chaykin LB, et al. The Efficacy of IDegLira (Insulin Degludec/Liraglutide Combination) in Adults with Type 2 Diabetes Inadequately Controlled with a GLP-1 Receptor Agonist and Oral Therapy: DUAL III Randomized Clinical Trial. Diabetes Ther. 2017;8(1):101-114.

54. Rodbard HW, Bode B, Harris S. IDegLira in insulin-naïve patients with type 2 diabetes (T2D) inadequately controlled on sulfonylureas (SU) alone or in combination with metformin: the DUAL IV study. Diabetes. 2015;64(Suppl 1):A255-A256.

55. Lingvay I, Pérez Manghi F, García-Hernández P, et al. Effect of Insulin Glargine Up-titration vs Insulin Degludec/Liraglutide on Glycated Hemoglobin Levels in Patients With Uncontrolled Type 2 Diabetes: The DUAL V Randomized Clinical Trial. JAMA. 2016;315(9):898-907.

56. Kiss I, Arold G, Roepstorff C, et al. Insulin degludec: pharmacokinetics in patients with renal impairment. Clin Pharmacokinet. 2014;53(2):175-83.

57. Kupcova V, Arold G, Roepstorff C, et al. Insulin degludec: pharmacokinetic properties in subjects with hepatic impairment. Clin Drug Investig. 2014;34(2):127-33.

58. Thalange $\mathrm{N}$, Deeb L, Iotova $\mathrm{V}$, et al. Insulin degludec in combination with bolus insulin aspart is safe and effective in children and adolescents with type 1 diabetes. Pediatr Diabetes. 2015;16(3):164-76.

59. Biester T, Danne T, Bläsig $\mathrm{S}$, et al. Pharmacokinetic and prandial pharmacodynamic properties of insulin degludec/insulin aspart in children, adolescents, and adults with type 1 diabetes. Pediatr Diabetes. 2016;17(8):642-649.

60. Ratner RE, Gough SCL, Mathieu C, et al. Hypoglycaemia risk with insulin degludec compared with insulin glargine in type 2 and type 1 diabetes: a pre-planned meta-analysis of phase 3 trials. Diabetes Obes Metab. 2013;15(2):175-84.

61. Onishi Y, Iwamoto Y, Yoo SJ, et al. Insulin degludec compared with insulin glargine in insulin- nave patients with type 2 diabetes: a 26week, randomized, controlled, Pan-Asian, treat-to-target trial. J Diabetes Investig. 2013;4(6):605-612.

62. Onishi Y, Ono Y, Rabøl R, et al. Superior glycaemic control with once-daily insulin degludec/insulin aspart versus insulin glargine in Japanese adults with type 2 diabetes inadequately controlled with oral drugs: a randomized, controlled phase 3 trial. Diabetes Obes Metab. 2013;15(9):826-832.

63. Sorli C, Warren M, Oyer D, et al. Elderly patients with diabetes experience a lower rate of nocturnal hypoglycaemia with insulin degludec than with insulin glargine: a meta-analysis of Phase IIIa trials. Drugs Aging. 2013;30(12):1009-1018.

64. Evans M, McEwan P, Foos V. Insulin degludec early clinical experience: does the promise from the clinical trials translate into clinical practice- a case-based evaluation. J Med Econ. 2015;18(2):96-105.

65. Home PD, Meneghini L, Wendisch U, et al. Improved health status with insulin degludec compared with insulin glargine in people with Type 1 diabetes. Diabet Med. 2012;29(6):716-720.

66. Ericsson A, Pollock RF, Hunt B, et al. Evaluation of the cost-utility of insulin degludec vs insulin glargine in Sweden. $J$ Med Econ. 2013;16(12):1442-1452.

67. Evans M, Wolden M, Gundgaard J, et al. Cost-effectiveness of insulin degludec compared with insulin glargine for patients with type 2 diabetes treated with basal insulin-from the UK health care cost perspective. Diabetes Obes Metab. 2014;16(4):366-75.

68. Evans M, Wolden M, Gundgaard J, et al. Cost-effectiveness of insulin degludec compared with insulin glargine in a basal-bolus regimen in patients with type 1 diabetes mellitus in the UK. J Med Econ. 2015;18(1):56-68.

69. Evans M, McEwan P. Clinical and cost-effectiveness of insulin degludec: from clinical trials to clinical practice. J Comp Eff Res. 2015;4:279-286.

70. Vora J, Christensen T, Rana A, et al. Insulin deglutec versus insulin glargine in type 1 and 2 diabetes mellitus: a meta-analisys. Diabetes Ther. 2014;5(2):435-446.

71. Goykhman S, Drincic A, Desmangles JC, et al. Insulin glargine: a review 8 year after its introduction. Expert Opin Pharmacother. 2009;10(4):705718.

72. Becker RH, Dahmen R, Bergmann K, et al. New insulin glargine 300 units $\bullet \mathrm{mL}-1$ provides a more even activity profile and prolonged glycemic control at steady state compared with insulin glargine 100 units $\bullet \mathrm{mL}-1$. Diabetes Care. 2015;38(4):637-643.

73. Dailey G, Lavernia F. A review of the safety and efficacy data for insulin glargine 300 units $/ \mathrm{ml}$, a new formulation of insulin glargine. Diabetes, Obes Metab. 2015:17(12):1107-1114.

74. Becker RH, Nowotny I, Teichert L, et al. Low within- and between-day variability in exposure to new insulin glargine $300 \mathrm{U} / \mathrm{ml}$. Diabetes Obes Metab. 2015;17(3):261-267.

75. Riddle MC, Bolli GB, Ziemen M, et al. New insulin glargine 300 units/ $\mathrm{mL}$ versus glargine 100 units $/ \mathrm{mL}$ in people with type 2 diabetes using basal and mealtime insulin: glucose control and hypoglycemia in a 6-month randomized controlled trial (EDITION 1). Diabetes Care. 2014;37(10):2755-2762.

76. Yki-Järvinen H, Bergenstal R, Ziemen M, et al. New insulin glargine 300 units $/ \mathrm{mL}$ versus glargine 100 units $/ \mathrm{mL}$ in people with type 2 diabetes using oral agents and basal insulin: glucose control and hypoglycemia in a 6-month randomized controlled trial (EDITION 2). Diabetes Care. 2014;37(12):3235-3243.

77. Bolli GB, Riddle MC, Bergenstal RM, et al. New insulin glargine $300 \mathrm{U} /$ $\mathrm{mL}$ compared with glargine $100 \mathrm{U} / \mathrm{mL}$ in insulin-naïve people with type 2 diabetes on oral glucose-lowering drugs: a randomized controlled trial (EDITION 3). Diabetes Obes Metab. 2015;17(4):386-394.

78. Matsuhisa M, Koyama M, Cheng X, et al. New insulin glargine $300 \mathrm{U} /$ $\mathrm{ml}$ versus glargine $100 \mathrm{U} / \mathrm{ml}$ in Japanese adults with type 1 diabetes using basal and mealtime insulin: glucose control and hypoglycaemia in a randomized controlled trial (EDITION JP 1). Diabetes Obes Metab. 2016;18(4):375-383. 
79. Terauchi Y, Koyama M, Cheng X, et al. New insulin glargine $300 \mathrm{U} / \mathrm{ml}$ versus glargine $100 \mathrm{U} / \mathrm{ml}$ in Japanese people with type 2 diabetes using basal insulin and oral antihyperglycaemic drugs: glucose control and hypoglycaemia in a randomized controlled trial (EDITION JP 2). Diabetes Obes Metab. 2016;18(4):366-74.

80. Home PD, Bergenstal RM, Bolli GB, et al. New Insulin Glargine 300Units/mL Versus Glargine 100 Units/mL in People With Type 1 Diabetes: A Randomized, Phase 3a, Open- Label Clinical Trial (EDITION 4). Diabetes Care. 2015;38(12):2217-2225.

81. Riddle MC, Yki-Järvinen H, Bolli GB, et al. One-year sustained glycaemic control and less hypoglycaemia with new insulin glargine $300 \mathrm{U} / \mathrm{ml}$ compared with $100 \mathrm{U} / \mathrm{ml}$ in people with type 2 diabetes using basal plus meal-time insulin: the EDITION 1 12-month randomized trial, including 6-month extension. Diabetes Obes Metab. 2015;17:835-842.

82. Yki-JärvinenH, Bergenstal RM, Bolli GB, et al. Glycaemic control and hypoglycaemia with new insulin glargine $300 \mathrm{U} / \mathrm{ml}$ versus insulin glargine $100 \mathrm{U} / \mathrm{ml}$ in people with type 2 diabetes using basal insulin and oral antihyperglycaemic drugs: the EDITION 2 randomized 12-month trial including 6-month extension. Diabetes Obes Metab. 2015:17(12):11421149 .
83. Ritzel R, Roussel R, Bolli GB, et al. Patient-level meta-analysis of EDITION 1, 2 and 3: glycaemic control and hypoglycaemia with new insulin glargine $300 \mathrm{U} / \mathrm{ml}$ versus glargine $100 \mathrm{U} / \mathrm{ml}$ in people with type 2 diabetes. Diabetes Obes Metab. 2015;17(9):859-867.

84. Brown MA, Courtney SD, Laurie WF. Role of Toujeo $\square$ in the treatment of diabetes mellitus. J Am Assoc Nurse Pract. 2016;28(9):503-9.

85. Toujeo (insulin glargine injection) U-300. Prescribing information; revised; 2015.

86. Steinstraesser A, Schmidt R, Bergmann K, et al. Investigational new insulin glargine $300 \mathrm{U} / \mathrm{ml}$ has the same metabolism as insulin glargine 100 U/ml. Diabetes Obes Metab. 2014;16(9): 873-876. 